\title{
Influences of Long-Term Memory-Guided Attention and Stimulus-Guided Attention on Visuospatial Representations within Human Intraparietal Sulcus
}

\author{
Maya L. Rosen, ${ }^{1}$ Chantal E. Stern, ${ }^{1,2,3}$ Samantha W. Michalka, ${ }^{3}$ Kathryn J. Devaney, ${ }^{1}$ and $\odot$ David C. Somers ${ }^{1,2,3}$ \\ ${ }^{1}$ Department of Psychological and Brain Sciences, ${ }^{2}$ Center for Memory and Brain, and ${ }^{3}$ Graduate Program for Neuroscience, Boston University, Boston, \\ Massachusetts 02215
}

Human parietal cortex plays a central role in encoding visuospatial information and multiple visual maps exist within the intraparietal sulcus (IPS), with each hemisphere symmetrically representing contralateral visual space. Two forms of hemispheric asymmetries have been identified in parietal cortex ventrolateral to visuotopic IPS. Key attentional processes are localized to right lateral parietal cortex in the temporoparietal junction and long-term memory (LTM) retrieval processes are localized to the left lateral parietal cortex in the angular gyrus. Here, using fMRI, we investigate how spatial representations of visuotopic IPS are influenced by stimulus-guided visuospatial attention and by LTM-guided visuospatial attention. We replicate prior findings that a hemispheric asymmetry emerges under stimulus-guided attention: in the right hemisphere (RH), visual maps IPS0, IPS1, and IPS2 code attentional targets across the visual field; in the left hemisphere (LH), IPS0-2 codes primarily contralateral targets. We report the novel finding that, under LTM-guided attention, both RH and LH IPS0-2 exhibit bilateral responses and hemispheric symmetry re-emerges. Therefore, we demonstrate that both hemispheres of IPS0-2 are independently capable of dynamically changing spatial coding properties as attentional task demands change. These findings have important implications for understanding visuospatial and memory-retrieval deficits in patients with parietal lobe damage.

Key words: fMRI; hemispheric asymmetry; memory retrieval; retinotopy

Significance Statement

The human parietal lobe contains multiple maps of the external world that spatially guide perception, action, and cognition. Maps in each cerebral hemisphere code information from the opposite side of space, not from the same side, and the two hemispheres are symmetric. Paradoxically, damage to specific parietal regions that lack spatial maps can cause patients to ignore half of space (hemispatial neglect syndrome), but only for right (not left) hemisphere damage. Conversely, the left parietal cortex has been linked to retrieval of vivid memories regardless of space. Here, we investigate possible underlying mechanisms in healthy individuals. We demonstrate two forms of dynamic changes in parietal spatial representations: an asymmetric one for stimulusguided attention and a symmetric one for long-term memory-guided attention.

\section{Introduction}

Human visual performance is superb, yet the capacity of human visual attention and short-term memory is limited to approximately four items. This paradox can be reconciled by recognizing

\footnotetext{
Received March 18, 2015; revised May 27, 2015; accepted July 10, 2015.

Author contributions: M.L.R., C.E.S., and D.C.S. designed research;M.L.R., S.W.M., and K.J.D. performed research; M.L.R., S.W.M., and K.J.D. analyzed data; M.L.R., C.E.S., and D.C.S. wrote the paper.

This work was supported by the National Institutes of Health (Grant R01EY022229 to D.C.S. and Grant

1F31MH101963-01 to S.W.M.). We thank Sam Ling and Summer Sheremata for helpful comments on this manuscript.

The authors declare no competing financial interests.

Correspondence should be addressed to David C. Somers, PhD, Department of Psychological and Brain Sciences,

Boston University, 64 Cummington Mall, Boston, MA 02215. E-mail: somers@bu.edu.

DOI:10.1523/JNEUROSCI.1055-15.2015

Copyright $\odot 2015$ the authors $\quad 0270-6474 / 15 / 3511358-06 \$ 15.00 / 0$
}

the important role that long-term memory (LTM) regularly plays in efficiently directing our attentional resources (for review, see Hutchinson and Turk-Browne, 2012). Human attentional performance is enhanced in familiar environments; however, the neural substrates of interactions between LTM and visual attention are understudied (Summerfield et al., 2006, 2011; Stokes et al., 2012; Rosen et al., 2015). Here, we examine how LTM-guided attention influences spatial representations in human parietal lobe and contrast this with the influences of stimulus-guided attention in the same task.

The human parietal lobe plays a central role in coding visuospatial information and multiple regions in the vicinity of the intraparietal sulcus (IPS) are known to contain maps of the con- 
tralateral visual field (Swisher et al., 2007; Silver and Kastner, 2009). These visuotopic maps exhibit a high degree of hemispheric symmetry when examined with standard fMRI retinotopic mapping analysis. In contrast to this symmetry, two forms of parietal lobe hemispheric asymmetry are well documented. A right hemisphere (RH) bias occurs for spatial attention. Prior studies identify the right temporoparietal junction (TPJ), a region that lies lateral to IPS, as the key structure in this attentional hemispheric bias (Mesulam, 1981; Corbetta and Shulman, 2011). A left hemisphere (LH) bias occurs in the angular gyrus (AnG) during successful LTM retrieval (Wagner et al., 2005; Vilberg and Rugg, 2007). Therefore, lateral portions of parietal lobe appear to exhibit hemispheric asymmetries, whereas the visuotopic maps along the medial bank of the IPS appear to be symmetric.

fMRI studies (Sheremata et al., 2010; Szczepanski et al., 2010; Sheremata and Silver, 2015) using demanding attention or shortterm memory tasks have found that the visuotopically mapped regions IPS0, IPS1, and IPS2 in the $\mathrm{RH}$, but not the $\mathrm{LH}$, dynamically change their spatial representations to code targets across the full visual field. The resulting hemispheric asymmetry in IPS spatial representations is consistent with key predictions of representational models of neglect (Heilman and Van Den Abell, 1980; Mesulam, 1981). However, these findings leave unanswered the question of what other cognitive factors might shape spatial representations in the parietal lobe.

Here, using a change-detection paradigm, we investigate the influences of LTM-guided and stimulus-guided attention on visuospatial representations within the IPS. We observe that each form of attentional guidance strongly influences IPS spatial representations. Stimulus-guided attention asymmetrically recruits $\mathrm{RH}$, but not LH, IPS for targets across the full visual field; under LTM-guided attention, hemispheric symmetry re-emerges in the IPS from a new source because both hemispheres exhibit robust bilateral responses. These two forms of dynamic changes in spatial representations occur with equivalent stimuli and therefore reflect top-down influences.

\section{Materials and Methods \\ Participants}

Twenty-two healthy participants (13 male, age 22-33 years) gave written informed consent (Boston University Institutional Review Board) before participating in a behavioral training session and two fMRI sessions (task, retinotopy). Two subjects were excluded due to weak retinotopic maps (see below).

\section{Experimental paradigms}

\section{Day 1: behavioral training}

Participants performed two behavioral tasks on day 1 intended to provide experience with the specific images to be used on day 2. A total of 216 outdoor scene images were used, 24 on change-detection LTM training (see below). Each participant viewed all scenes and scenes were counterbalanced between conditions across the participant pool. An altered version of each scene was created using Adobe Photoshop (e.g., removed tree, added window).

Change-detection encoding task. Participants were shown 24 scenes in a change-detection flicker paradigm (Rosen et al., 2014) with the goal of training participants on these images for the LTM-guided condition on day 2. On each trial, a scene appeared $(1000 \mathrm{~ms})$, followed by a blank screen $(250 \mathrm{~ms})$, the altered scene image $(1000 \mathrm{~ms})$, and a blank screen $(250 \mathrm{~ms})$ in a loop for $15 \mathrm{~s}$. Participants were instructed to mouse-click on the change. Each trial ended with a $10 \mathrm{~s}$ "reveal period" in which original and altered scenes alternated (without blanks), to reinforce the scene change location (for further details on training efficacy, see Rosen et al., 2014).
Man-made/natural judgment encoding task. Participants viewed 192 scene images for $3000 \mathrm{~ms}$ each (no image changes) and reported whether the scene was primarily natural or man-made. This exposure served to familiarize participants with the scenes, but not the changes, that would be used in the stimulus-guided attention condition on day 2 .

\section{Day 2: test}

fMRI scanning occurred $24-48 \mathrm{~h}$ after day 1 training. We contrasted LTM-guided attention and stimulus-guided attention in left and right visual fields (LVF and RVF, respectively), along with control conditions. Trials were presented in 12 counterbalanced blocks per run (2 LTMguided: left cue, 2 LTM-guided: right cue, 2 stimulus-guided: left cue, 2 stimulus-guided: right cue, 1 no-cue: left, 1 no-cue: right, 2 passive). Blocks contained a $1 \mathrm{~s}$ block-cue and $65.9 \mathrm{~s}$ trials. Each participant performed 8 runs.

Participants performed a "single-shot" change detection task under different cueing conditions (Fig. 1). The initial scene appeared (3000 $\mathrm{ms})$, followed by a blank gray screen $(250 \mathrm{~ms})$ and then either the original or altered scene appeared $(150 \mathrm{~ms})$ and finally was replaced by a blank screen for the remainder of each trial $(2500 \mathrm{~ms})$ while responses were collected. The $150 \mathrm{~ms}$ probe presentation was chosen to prevent subjects from moving their attentional focus once the probe appeared. Image changes occurred on $50 \%$ of the trials. Participants reported whether a change occurred. Scenes subtended $\sim 12^{\circ} \times$ $8^{\circ}$ of visual angle. Changes did not occur within $0.5^{\circ}$ of fixation or within $0.5^{\circ}$ of scene edges.

Participants were instructed to fixate centrally and covertly attend to the cued scene location. In the LTM-guided attention condition, participants directed attention based on learned spatial location; that is, LTM was the only source of cueing information. In the stimulus-guided attention condition, participants directed attention to an exogenously cued (red and white square outline, $\sim 1.3^{\circ} \times 1.3^{\circ}$ of visual angle presented for the first $1500 \mathrm{~ms}$ of a trial) spatial location in scenes (Fig. 1). In the no-cue condition, participants were instructed to actively attend to the whole novel scene; in the passive condition, participants simply made a button press to each (novel) scene. The no-cue condition, which was included as a behavioral control to demonstrate the utility of LTM and stimulus cues, lacked spatial cueing information and was analyzed only as regressors of no interest. The LTM-guided condition scenes were repeated once per run (eight times total). All other images were only presented once for each participant.

Eye movements. Participants were instructed to maintain central fixation throughout the experiment during fMRI scanning. Eye position was monitored via video camera for all subjects and eye movements in excess of $2^{\circ}$ of visual angle were recorded. For 3 participants ( 2 male, 1 female), 1 run was excluded from fMRI analysis due to excessive ( $>5 \%$ of trials) eye movements during that run. Otherwise, participants overall maintained fixation on $99.0 \%$ of trials (stimulus-guided: $98.3 \%$, LTM-guided: $99.3 \%$; no-cue $=99.1 \%$; passive $=99.2 \%)$. We cannot rule out the possible influence of eye micromovements $\left(<2^{\circ}\right)$; however, to affect the results, these would need to differ between target hemifields for one form of attention and not for the other.

fMRI data acquisition. $\mathrm{fMRI}$ data were acquired using a 3 tesla Siemens TIM Trio with 32-channel head coil at Harvard University (Cambridge, Massachusetts). Functional scans were acquired using T2*-weighted, gradient echo, echoplanar images $(\mathrm{TR}=2.6 \mathrm{~s}$; $\mathrm{TE}=30 \mathrm{~ms}$; voxel size $3.1 \times 3.1 \times 3.0 \mathrm{~mm} ; 42$ slices with $0 \%$ gap, yielding full cerebral cortical coverage). Functional data were aligned with high-resolution $(1.0 \times$ $1.0 \times 1.3 \mathrm{~mm})$ T1-weighted images. For 14 participants, the highresolution structural images were acquired at the same facility; for eight participants, they were acquired on an identically equipped scanner and coil at the Martinos Center for Biomedical Imaging (Charlestown, Massachusetts).

$f M R I$ data analysis. For each participant, the cortical surface of each hemisphere was computationally reconstructed from the highresolution anatomical volume using FreeSurfer software (Fischl, 2012). fMRI task data were analyzed using the FreeSurfer 5.1.0 software package. Intensity normalization and motion correction were performed before signal averaging. Cortical surface and region of interest (ROI) 


\section{A Stimulus-guided}

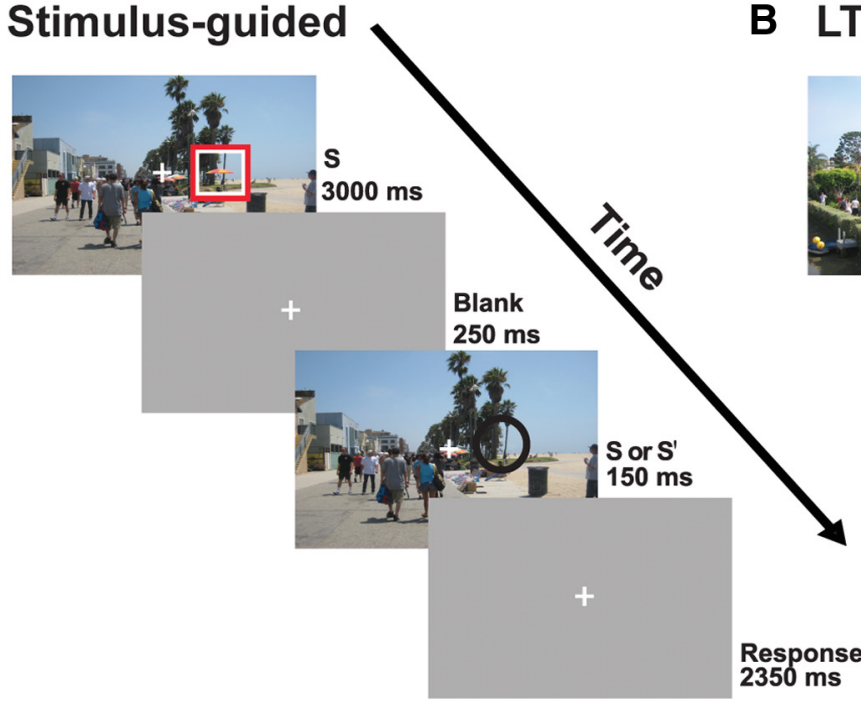

B LTM-guided

$2350 \mathrm{~ms}$

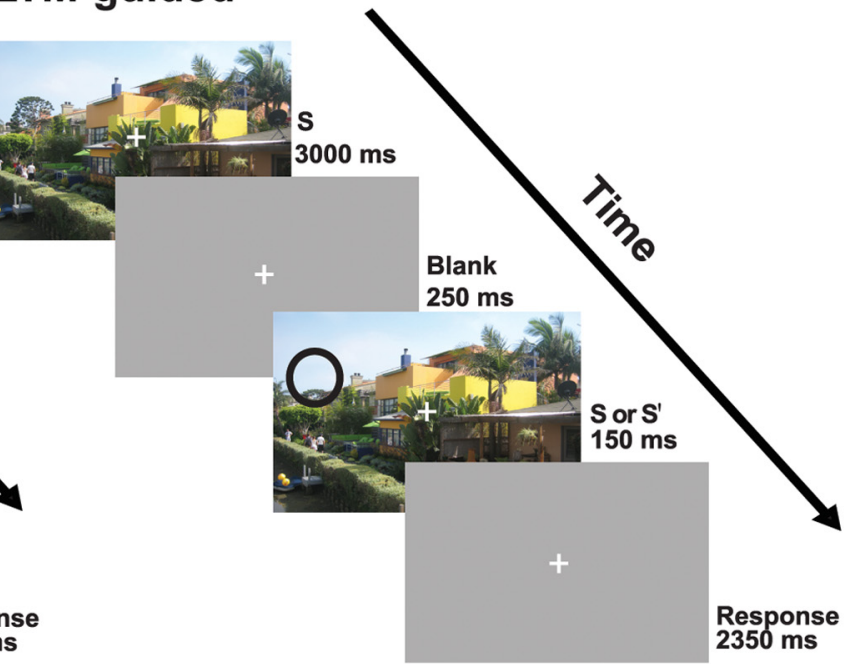

Figure 1. One-shot change detection paradigms. A scene (S) was presented for $3000 \mathrm{~ms}$, followed by a blank screen ( $250 \mathrm{~ms})$, a very brief presentation (150 ms) of either an identical or altered image (Sor S'), and another blank screen $(2350 \mathrm{~ms})$. Participants held central fixation while reporting whether a change occurred. $A$, Stimulus-guided condition. Participants viewed scenes that they had previously studied without scene changes. A red and white square explicitly cued (for $1500 \mathrm{~ms}$ ) the location of the potential scene change and then disappeared. $\boldsymbol{B}$, LTM-guided condition. Participants covertly attended to the remembered location of the potential change and no explicit spatial cue was provided.

A

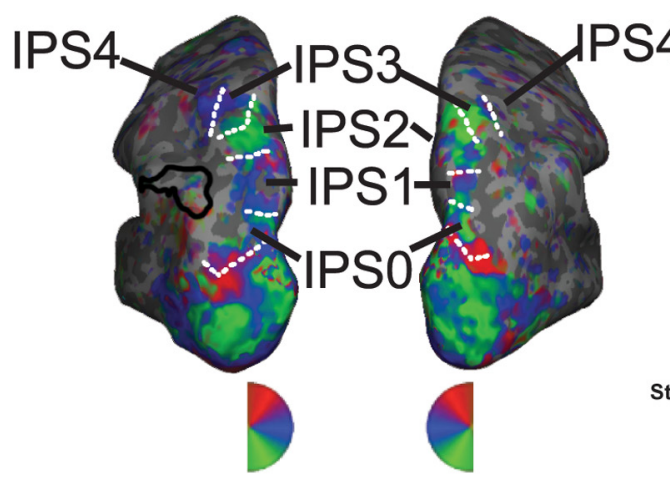

B

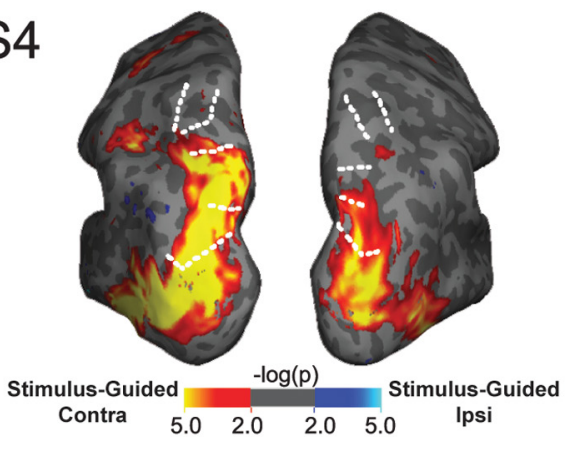

C

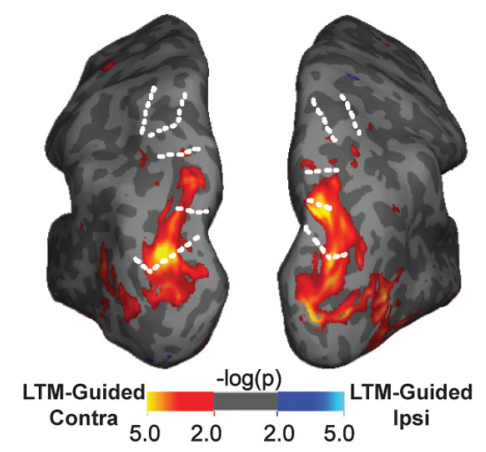

Figure 2. Activation maps for retinotopic mapping, stimulus-guided, and LTM-guided attention. $A$, Retinotopic maps of visual polar angle representation in IPS0-IPS4 from a representative subject. IPS maps lie medial to the lateral parietal region that exhibits greater activation for LTM-guided attention than for stimulus-guided attention (black outline; Rosen et al., 2015). B, C, Group average of activation in response to contralateral versus ipsilateral targets in stimulus-guided and LTM-guided attention, respectively.

analyses were performed using a general linear model (GLM) with regressors that matched the time course of all task conditions.

Cortical surface analysis. Single-participant fMRI data were registered to an average cortical surface space (FreeSurfer "fsaverage" brain). Data were analyzed for each vertex using a GLM with each condition as a predictor. Three motion correction regressors were included. The BOLD signal was modeled as a linear, time-invariant system with $\gamma$ response function assumed for each condition with a delay $\delta=2.25$ and a delay time constant $\tau=1.25$. Random-effects group analyses were performed using surface-based averaging techniques (Fischl, 2012). A $t$ test was performed for each vertex to compare differences in activation between conditions and $p$-values were projected onto the cortical surface. Previously, using this dataset, we reported an analysis of the contrast between LTM-guided attention and stimulus-guided attention that ignored the location of attention (Rosen et al., 2015); notably, a region in lateral parietal cortex, on the lateral bank of the IPS, and extending into the AnG, that exhibited stronger activation for LTM-guided attention than for stimulus-guided attention lies lateral to visuotopic IPS (Fig. 2A, black outline).

Retinotopic mapping and ROI definition. To map the visual field representations within the IPS, we used the phase-encoded protocol described in Swisher et al. (2007) using Freesurfer 4.0.2. A flickering chromatic radial wedge (polar angle) checkerboard swept around a fixation point at the center of the screen at a periodicity of $55.46 \mathrm{~s}$ (12 cycles per $665.6 \mathrm{~s}$ run). Subjects fixated at a center fixation point and made a button press when the fixation point dimmed at random throughout the run. The phase of the response at each vertex was used to determine its preferred polar angle (Figs. 2A, 3A). ROIs for areas IPS0, IPS1, IPS2, IPS3, and IPS4 were drawn based on reversals in the polar angle phases (Swisher et al., 2007; Fig. 2A). ROIs were then restricted to those vertices that were significantly activated ( $p<0.05$, uncorrected) by retinotopy. For two subjects, the five IPS regions could not be drawn using this statistical threshold and therefore these subjects were dropped from all analyses.

Percent signal change was extracted for each ROI for each contrast compared with passive viewing. Each hemisphere was analyzed for each contralateral and ipsilateral visual field conditions. Contralateral bias in each IPS region for each condition (LTM-guided and stimulus-guided) was quantified as a contralateral index (CI) as follows:

$$
C I=\frac{(\text { Contra }- \text { Ipsi) }}{\sqrt{\sigma_{\text {Contra }}+\sigma_{\text {Ipsi }}}}
$$


A

Left Hemisphere

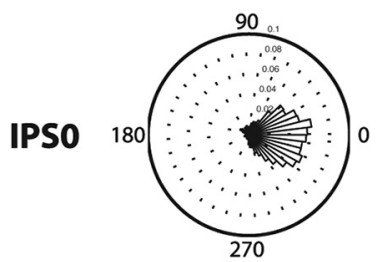

Right Hemisphere
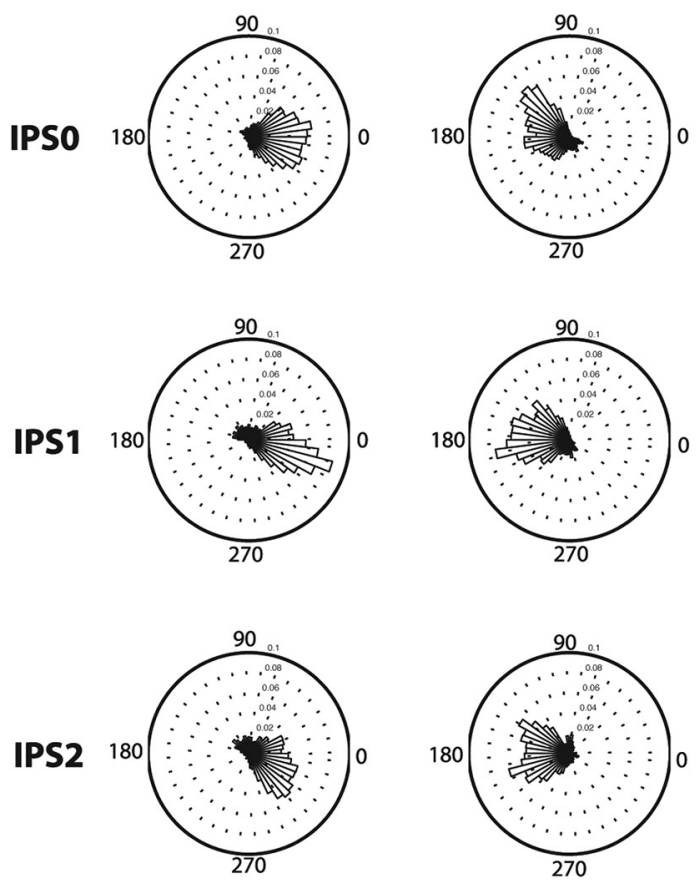

B
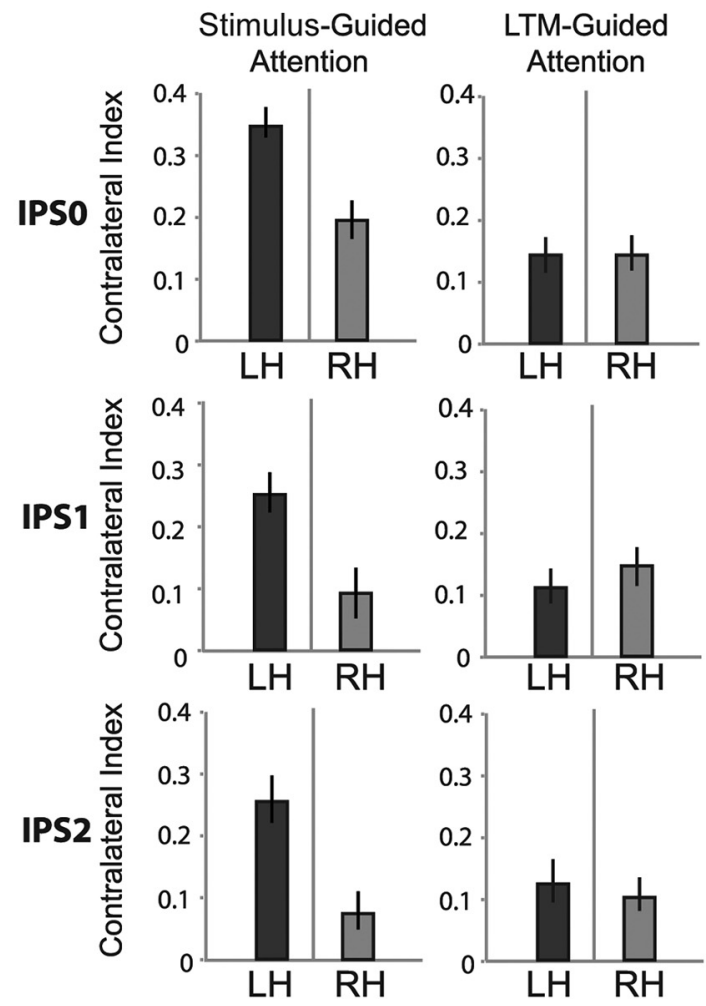

Figure 3. Comparison of contralateral bias during visuotopic mapping, stimulus-guided, and LTM-guided attention. $A$, Preferred visual polar angle for vertices activated during mapping in left and right IPSO to IPS2. B, C, Contralateral bias for each IPS region ( $0-2)$ for stimulus-guided ( $\boldsymbol{B})$ and LTM-guided $(\boldsymbol{C})$ attention in LH and RH.

Where Contra and Ipsi refer to the percent signal change for targets in the contralateral and ipsilateral visual fields, respectively, for a hemisphere ROI. $\sigma$ is the variance in a condition over all runs and was used to normalize contralaterality effects as a form of signal-to-noise measurement and to avoid the "vanishing denominator" problem that can occur when spatial attention suppresses ipsilateral responses below baseline.

\section{Results}

\section{Behavioral performance}

Subjects performed well both in LTM-guided attention and stimulus-guided attention conditions and in both visual hemifields. A Condition $\times$ Visual Field ANOVA revealed no significant main effect of condition, visual field, or interaction (all $p>$ 0.1 ; stimulus-guided LVF $d^{\prime}=2.52 \pm 0.34$, stimulus-guided RVF $d^{\prime}=2.37 \pm 0.58, \mathrm{LTM}$-guided LVF $=2.72 \pm 0.81$, LTM-guided $\mathrm{RVF}=2.56 \pm 0.69)$.

\section{fMRI results}

Retinotopic mapping was performed in each subject and, as expected, revealed multiple maps of the contralateral visual field within the IPS of each hemisphere. Both right and left IPS exhibited a strong bias for stimuli in the contralateral visual field and these patterns appeared to be highly symmetric (Fig. 2A). Note that the visuotopic IPS regions lie dorsomedial to the parietal regions that exhibit overall greater activation for LTM-guided attention relative to stimulus-guided attention (lateral IPS/AnG; Fig. 2A, black outlines, replotted from Rosen et al., 2015).

In the attention task scans, fMRI activation in each hemisphere was contrasted between contralateral and ipsilateral visual field target stimuli. A strong hemispheric asymmetry was observed within the IPS for the stimulus-guided attention condition (Fig. 2B). LH IPS exhibits a strong contralateral bias, with weak responses for ipsilateral targets. In contrast, RH IPS exhibits strong responses for both ipsilateral and contralateral targets and thus has a diminished contralateral bias.

Hemispheric symmetry re-emerges in the IPS during the LTM-guided attention condition. Both left and right IPS responded strongly to both ipsilateral and contralateral targets and thus both hemispheres exhibited weaker contralateral biases (Fig. $2 C)$. Therefore, both LTM-guided and stimulus-guided attention alter the spatial extent of visual field coding within the IPS; moreover, the two forms of visuospatial attention guidance produce different changes in the IPS spatial representations.

To quantify these attention-driven dynamic changes in the visual field representations within the IPS, an ROI analysis was performed for retinotopically mapped IPS0, IPS1, IPS2, IPS3, and IPS4 in each hemisphere of each subject. This analysis was restricted to vertices that exhibited significant activation during retinotopic mapping (see Materials and Methods). We performed three-factor (Hemisphere $\times$ Condition $\times$ Visual Field) ANOVAs for each IPS region. The critical test for differences in hemispheric asymmetry between the LTM-guided and stimulusguided attention conditions is a three-way interaction among hemisphere, attentional condition, and visual hemifield. IPS0, IPS1, and IPS2 all displayed this significant interaction (Table 1, all $p<0.05$, uncorrected), whereas IPS 3 and IPS4 did not (Table $1, p<0.2$ ). In IPS0, the significance of the three-way interaction in IPS0 does not survive correction for multiple comparisons. However, consistent with prior reports of an attention-driven hemispheric asymmetry in this region (Sheremata et al., 2010; Sheremata and Silver, 2015), our follow-up analysis revealed a significant difference in $\mathrm{CI}$ in the stimulus-guided condition. In contrast, we observed near perfect symmetry between the hemi- 
Table 1. Activation within left and right IPSO -IPS4 during LTM-guided and STIM-guided attention

\begin{tabular}{|c|c|c|c|c|c|c|c|c|c|c|}
\hline \multirow[b]{3}{*}{ Region } & \multicolumn{4}{|l|}{ LH } & \multicolumn{4}{|l|}{ RH } & \multirow[b]{3}{*}{ F-statistic } & \multirow{3}{*}{$\begin{array}{l}p \text {-value } \\
\text { (uncorrected) }\end{array}$} \\
\hline & \multicolumn{2}{|l|}{ LTM-guided } & \multicolumn{2}{|l|}{ STIM-guided } & \multicolumn{2}{|l|}{$\underline{\text { LTM-guided }}$} & \multicolumn{2}{|l|}{$\underline{\text { STIM-guided }}$} & & \\
\hline & Contralateral & Ipsilateral & Contralateral & Ipsilateral & Contralateral & Ipsilateral & Contralateral & Ipsilateral & & \\
\hline IPS1 & $0.32 \pm 0.05$ & $0.23 \pm 0.06$ & $0.49 \pm 0.05$ & $0.30 \pm 0.05$ & $0.31 \pm 0.05$ & $0.20 \pm 0.04$ & $0.48 \pm 0.06$ & $0.42 \pm 0.04$ & 11.40 & 0.003 \\
\hline IPS2 & $0.41 \pm 0.05$ & $0.34 \pm 0.05$ & $0.58 \pm 0.06$ & $0.40 \pm 0.04$ & $0.43 \pm 0.05$ & $0.34 \pm 0.05$ & $0.56 \pm 0.05$ & $0.50 \pm 0.05$ & 14.05 & 0.001 \\
\hline IPS3 & $0.36 \pm 0.07$ & $0.32 \pm 0.06$ & $0.48 \pm 0.05$ & $0.38 \pm 0.05$ & $0.31 \pm 0.06$ & $0.26 \pm 0.06$ & $0.46 \pm 0.07$ & $0.42 \pm 0.07$ & 1.13 & 0.302 \\
\hline
\end{tabular}

Percent signal change (vs passive) within left and right retinotopically mapped IPSO-4 during LTM-guided and stimulus-guided attention to the contralateral and ipsilateral visual field. F-statistics and $p$-values refer to the significance of the three-way (Hemisphere $\times$ Condition $\times$ Visual Field) interaction.

spheres in the LTM-guided condition (see below and Fig. 3B), thus demonstrating the re-emergence of hemispheric symmetry in IPS0. The main effect of Condition was significant in all IPS regions (IPS0-4), such that activation was greater in the stimulusguided condition than the LTM-guided condition (all $p<$ 0.0001 ) and none of the regions showed a main effect of Hemisphere (all $p>0.05)$.

The attention-driven dynamic changes in visual field coding within IPS0, IPS1, and IPS2 are summarized in Figure 3. The distribution of preferred visual field polar angles reveal a very robust bias for stimuli presented in the contralateral visual field under retinotopic mapping and this bias is highly symmetric between hemispheres (Fig. 3A). To quantify contralateral biases in LTM-guided and stimulus-guided attention, we computed a CI for IPS0, IPS1, and IPS2 in each hemisphere (see Materials and Methods). The CI is significantly greater than 0 in both conditions across all regions and both hemispheres (all $p<0.05$, corrected). However, all three regions showed a significantly stronger $\mathrm{CI}$ in stimulus-guided attention in the LH compared with the RH (all $p<0.05$, corrected Fig. $3 B$ ) and none of the regions showed a significant difference in CI between hemispheres in the LTM-guided attention condition (all $p>0.4$; Fig. $3 C$ ). In each region, the CI was significantly stronger in the $\mathrm{LH}$ for stimulus-guided attention than for LTM-guided attention (all $p<0.05$, corrected). In the $\mathrm{RH}$, there was no difference in the $\mathrm{CI}$ between LTM-guided attention and stimulus-guided attention in any ROI (all $p>0.1)$.

\section{Discussion}

These results demonstrate two forms of attentionally driven changes in spatial representations within human IPS. Under simple stimulus conditions, spatial coding is strongly biased to the contralateral visual field and highly symmetric between the hemispheres. Under stimulus-guided attention conditions, a hemispheric asymmetry emerges because the right IPS0-2 responds robustly to stimuli in both visual hemifields, whereas the left IPS0-2 maintains a strong contralateral bias. In contrast, during LTM-guided attention, hemispheric symmetry re-emerges because both left and right IPS0-2 respond robustly to targets in both visual hemifields, resulting in a substantially reduced contralateral bias. The dynamic changes under stimulus-guided attention replicate prior findings (Sheremata et al., 2010; Szczepanski et al., 2010; Sheremata and Silver, 2015). The dynamic changes under LTM-guided attention are novel. Together, our results demonstrate that spatial representations within the IPS in each hemisphere are strongly and differentially influenced by cognitive factors.

Prior work has demonstrated that a region in left AnG is specifically and asymmetrically recruited during memory retrieval (Wagner et al., 2005; Vilberg and Rugg, 2007). This memory- retrieval-related activation lies ventrolateral to the visual maps in the IPS (Fig. 2A, black lines, replotted from Hutchinson et al., 2014; Rosen et al., 2015). Therefore, the observed changes in spatial responsiveness in left IPSO-2 cannot simply be attributed to nonspatial-memory-retrieval mechanisms within the left visuotopic IPS. Rather, our results suggest that memory retrievalrelated processes dynamically widen the range of spatial representations within the left IPS0-2. Interestingly, there is a parallel set of observations in the $\mathrm{RH}$. The preferential role for the right parietal lobe in visuospatial attention is a well-established hemispheric asymmetry, but the primary source of this asymmetry lies ventrolateral to the visuotopic maps of IPS in the vicinity of the TPJ, not in the maps themselves (Corbetta and Shulman, 2011). Therefore, two complementary forms of hemispheric asymmetries each appear to increase dynamically the extent of spatial representation within visuotopically mapped IPS. For technical reasons, we were unable to compare directly the degree of contralaterality under retinotopic mapping with our two attentional conditions; however, a recent study has demonstrated that the right IPS increases its extent of spatial representation in a stimulus-directed attention paradigm relative to a neutral attention condition (Sheremata and Silver, 2015). Our results are consistent with that finding and further indicate that the extent of $\mathrm{LH}$ representations also expands under LTM-guided attention.

The stimulus-guided condition included a peripheral cue stimulus (red box) that was not present in the LTM-guided condition. It is unlikely that this cue is critical to the hemispheric asymmetry observed in the stimulus-guided condition. This cue was presented symmetrically across trials and several previous studies have observed a hemispheric asymmetry for attention and visual short-term memory in the IPS in the absence of any explicit visual cue (Hopfinger et al., 2010; Sheremata et al., 2010; Szczepanski et al., 2010; Sheremata et al., 2015). Overall, BOLD responses were greater in the stimulus-guided than the LTMguided condition. This difference might reflect the effect of the cue stimulus in the stimulus-guided condition and/or diminished responses due to repetition of the stimuli in the LTMguided condition; however, these overall response differences do not affect the differences observed in the spatial responsiveness of IPS maps.

Hemispatial neglect syndrome, which almost always manifests as left hemifield neglect subsequent to RH damage (Heilman and Van Den Abell, 1980; Mesulam, 1981), is strongly associated with damage at the border of the right parietal and temporal lobes in the TPJ, far lateral to IPS visuotopy. Representational models of neglect suggest a parietal lobe hemispheric asymmetry in which the LH codes only contralateral space, whereas the $\mathrm{RH}$ codes the full visual field (Heilman and Van Den Abell, 1980; Mesulam, 1981). The present work and other neuroimaging 
studies demonstrate that, under stimulus-guided attention, this pattern emerges dynamically in the IPS even though retinotopy alone reveals symmetric contralateral representations (Hopfinger et al., 2010; Sheremata et al., 2010; Szczepanski et al., 2010; Sheremata and Silver, 2015). A key neuroimaging study of neglect patients also points to changing spatial codes in the IPS; the visuotopic maps in the IPS become unbalanced in neglect patients and slowly rebalance in patients who recover from neglect (Corbetta et al., 2005).

Our findings are consistent with evidence that neglect persists when patients create a mental image using LTM (Bisiach and Luzzatti, 1978). Mental imagery requires both retrieval and attentional inspection of mnemonic images. With the $\mathrm{LH}$ parietal lobe intact, these patients could retrieve mnemonic images; however, with damage to the RH parietal lobe, they are not able to access attentionally the left side of these remembered scenes. A recent behavioral study in neglect patients found visuospatial asymmetries for short-term memory, but not LTM (Moreh et al., 2014).

Episodic memory-retrieval deficits occur in patients with ventral parietal lobe damage (Berryhill et al., 2011) and the data suggest that LTM retrieval mechanisms are less lateralized in the ventral parietal lobe than are spatial attention mechanisms. In our study, LTM-guided attention yielded symmetric changes to IPS0-2 spatial representations in both hemispheres. A parametric study is required to identify the relative contributions of mnemonic and attentional processes to spatial representational changes.

LTM makes important contributions to the efficient deployment of our very limited attentional resources. The present study advances our understanding of the neural substrates that support interactions between LTM and visuospatial attention. The dissociation of spatial representations observed here parallels functional dissociations in more lateral regions of parietal cortex (the right TPJ and left AnG), suggesting important avenues for further linking the functions of these regions and informing our understanding of the nature of visuospatial and memory-retrieval deficits in patients with parietal lobe damage.

\section{References}

Berryhill ME, Chein J, Olson IR (2011) At the intersection of attention and memory: the mechanistic role of the posterior parietal lobe in working memory. Neuropsychologia 49:1306-1315. CrossRef Medline

Bisiach E, Luzzatti C (1978) Unilateral neglect of representational space. Cortex 14:129-133. CrossRef Medline

Corbetta M, Shulman GL (2011) Spatial neglect and attention networks. Annu Rev Neurosci 34:569-599. CrossRef Medline

Corbetta M, Kincade MJ, Lewis C, Snyder AZ, Sapir A (2005) Neural basis and recovery of spatial attention deficits in spatial neglect. Nat Neurosci 8:1603-1610. CrossRef Medline
Fischl B (2012) FreeSurfer. Neuroimage 62:774-781. CrossRef Medline

Heilman KM, Van Den Abell T (1980) Right hemisphere dominance for attention: the mechanism underlying hemispheric asymmetries of inattention (neglect). Neurology 30:327-330. CrossRef Medline

Hopfinger JB, Camblin CC, Parks EL (2010) Isolating the internal in endogenous attention. Psychophysiology 47:739-747. Medline

Hutchinson JB, Turk-Browne NB (2012) Memory-guided attention: control from multiple memory systems. Trends Cogn Sci 16:576-579. CrossRef Medline

Hutchinson JB, Uncapher MR, Weiner KS, Bressler DW, Silver MA, Preston AR, Wagner AD (2014) Functional heterogeneity in posterior parietal cortex across attention and episodic memory retrieval. Cereb Cortex 24: 49-66. CrossRef Medline

Mesulam MM (1981) A cortical network for directed attention and unilateral neglect. Ann Neurol 10:309-325. CrossRef Medline

Moreh E, Malkinson TS, Zohary E, Soroker N (2014) Visual memory in unilateral spatial neglect: immediate recall versus delayed recognition. J Cogn Neurosci 26:2155-2170. CrossRef Medline

Rosen ML, Stern CE, Somers DC (2014) Long-term memory guidance of visuospatial attention in a change-detection paradigm. Front Psychol 5:266. Medline

Rosen ML, Stern CE, Michalka SW, Devaney KJ, Somers DC (2015) Cognitive control network contributions to memory-guided visual attention. Cereb Cortex. In press.

Sheremata SL, Silver MA (2015) Hemisphere-dependent attentional modulation of human parietal visual field representations. J Neurosci 35:508517. CrossRef Medline

Sheremata SL, Bettencourt KC, Somers DC (2010) Hemispheric asymmetry in visuotopic posterior parietal cortex emerges with visual short-term memory load. J Neurosci 30:12581-12588. CrossRef Medline

Silver MA, Kastner S (2009) Topographic maps in human frontal and parietal cortex. Trends Cogn Sci 13:488-495. CrossRef Medline

Stokes MG, Atherton K, Patai EZ, Nobre AC (2012) Long-term memory prepares neural activity for perception. Proc Natl Acad Sci U S A 109: E360-E367. CrossRef Medline

Summerfield JJ, Lepsien J, Gitelman DR, Mesulam MM, Nobre AC (2006) Orienting attention based on long-term memory experience. Neuron 49: 905-916. CrossRef Medline

Summerfield JJ, Rao A, Garside N, Nobre AC (2011) Biasing perception by spatial long-term memory. J Neurosci 31:14952-14960. CrossRef Medline

Swisher JD, Halko MA, Merabet LB, McMains SA, Somers DC (2007) Visual topography of human intraparietal sulcus. J Neurosci 27:5326-5337. CrossRef Medline

Szczepanski SM, Konen CS, Kastner S (2010) Mechanisms of spatial attention control in frontal and parietal cortex. J Neurosci 30:148-160. CrossRef Medline

Vilberg KL, Rugg MD (2007) Dissociation of the neural correlates of recognition memory according to familiarity, recollection, and amount of recollected information. Neuropsychologia 45:2216-2225. CrossRef Medline

Wagner AD, Shannon BJ, Kahn I, Buckner RL (2005) Parietal lobe contributions to episodic memory retrieval. Trends Cogn Sci 9:445-453. CrossRef Medline 